\title{
A resource block organization strategy for scheduling in carrier aggregated systems
}

\author{
Guillermo Galaviz ${ }^{1,2^{*}}$, David H Covarrubias ${ }^{1}$, Angel G Andrade ${ }^{2}$ and Salvador Villarreal ${ }^{1}$
}

\begin{abstract}
Carrier aggregation (CA) is a promising technology that will allow IMT-Advanced system candidates to achieve spectrum bandwidths of up to $100 \mathrm{MHz}$ using available system resource blocks (RB), even if these are fragmented. Implementation of CA functionality is managed through the use of schedulers capable of assigning multiple RBs to a user. When each available RB is handled individually, the delay from assigning multiple RBs to each user can affect the quality of service (QoS). In this article we develop an efficient scheduling strategy to reduce spectrum resource assignment delay in systems that make use of CA. This strategy is based on an a-priory organization of available RBs in sets. Two different RB Organization Algorithms are compared. In order to evaluate the performance of the proposed strategy numerical simulation was performed using a Round Robin scheduler for the downlink of a macro-cellular environment. Results show that using the proposed strategy it is possible to reduce the delay required to assign resources to users without affecting the downlink user capacity when compared to block by block scheduling strategies proposed in literature. The benefits of using the proposed strategy are discussed as well as improvement opportunities.
\end{abstract}

\section{Introduction}

Wireless cellular communication systems have been part of our everyday life for more than 30 years. Currently, wireless cellular systems are evolving from voice oriented solutions into broadband wireless access systems. Recent developments of next generation wireless cellular systems in the IMT Advanced initiative specify a 1 Gbps downlink data rate for static users and $100 \mathrm{Mbps}$ for high mobility users. In order to achieve such high data rates in a wireless system, spectrum efficiency has become a physical layer design priority. Technology developments such as MIMO-OFDM together with high order modulation schemes and efficient error correcting codes, allow for a spectrum efficiency of up to $15 \mathrm{bps} / \mathrm{Hz}$ [1]. However, even with such high spectrum efficiency (achieved only under optimum channel conditions) there is a large requirement of spectrum bandwidth. For the $1 \mathrm{Gbps}$ transfer rate and a spectrum efficiency of $15 \mathrm{bps} / \mathrm{Hz}$, approximately $67 \mathrm{MHz}$ of bandwidth would be required by one user during enough time to complete a data transfer.

\footnotetext{
*Correspondence: galavizy@cicese.edu.mx

'Department of Electronics and Communications, CICESE Research Center, Ensenada, Mexico

Full list of author information is available at the end of the article
}

Current versions of broadband wireless systems make use of channel bandwidths of up to $20 \mathrm{MHz}$ [2]. Therefore, a different spectrum management scheme is required for next generation wireless systems in order to provide the required bandwidth. Due to the fragmentation of the spectrum bands for next generation broadband wireless cellular systems, the expected growth of broadband wireless users, and the large bandwidths required to provide high data rate services [3], the spectrum available is considered to be scarce and fragmented.

Carrier aggregation (CA) has been defined as an enabling technology to overcome the spectrum scarcity and fragmentation problem. CA allows a system to aggregate multiple spectrum resources (resource blocks or RBs) and assign them to a single user in order to provide the sufficient bandwidth for a given service. CA works by allowing the system to assign RBs that may or may not be contiguous and considering the possibility that they are in different frequency bands. This derives in three different types of CA [4]:

- Contiguous CA: Aggregation of contiguous RBs within the same frequency band.

\section{Springer}


- Non-contiguous intra-band CA: Aggregation of non-contiguous RBs available within the same frequency band.

- Non-contiguous inter-band CA: Aggregation of non-contiguous RBs available in different frequency bands.

In order to implement CA spectrum assignment to a single user, a scheduler with multiple RB assignment capabilities is required by the system. In general, the task of the scheduler will be to optimize resource usage in a feasible amount of time. Resource usage can be measured as the throughput handled by the network. However, due to the quality of service (QoS) that has to be offered, it has to be possible to every user to make use of the available resources regardless of the achievable throughput. This characteristic is referred to as fairness. From the network perspective, there will always be a tradeoff between throughput and fairness.

Some of the scheduling proposals for CA systems available in literature involve an adaptation of algorithms used in non-CA systems, such as Proportional Fair and Processor Sharing. The way they provide CA capabilities is by scheduling each available RB individually. When all the resources required by a user are scheduled, the user has enough resources (bandwidth) to be serviced. Proposals in [5-7] deal with CA scheduling using this strategy, which we refer to as Block by Block Scheduling. A different strategy is presented in [8], where a water filling algorithm is used to provide CA capabilities. A novel approach for CA is presented in [9], where a technique called separated burst-level scheduling (SBLS) is implemented using a two level scheduler structure.

Since the number of RBs required by next generation wireless cellular systems can be quite large, the time required by a scheduler to assign all the resources needed by a user can become considerably high if RBs are handled individually. This will yield in a potentially excessive delay of the scheduling tasks. This operation time will actually depend on the organization of the spectrum resources that the scheduler can handle. Delay is an important aspect that has to be considered within IMT-Advanced system candidates. In order to fulfill the QoS goals of high data rates, low latency and high user capacity for demanding applications such as high definition real time video an achievable user plane packet delay of $2 \mathrm{~ms}$ is defined [1]. This packet delay involves the time epoch at which a packet arrives at the scheduler queue until it is completely transmitted in an unloaded system. Achieving this goal involves an improvement of several subsystems. One important component of the packet delay comes from the scheduling process. Efficient scheduling of available resources is typically characterized by higher delays, while simple scheduling algorithms usually waste system resources. Given the delay restrictions established for IMTAdvanced system candidates such as LTE-Advanced, it is important to consider the delay when designing schedulers with CA capabilities.

In this article we present a scheduling strategy based on the assignment of pre-organized RB sets. We will refer to this strategy as Set Scheduling. The main idea behind Set Scheduling was presented in [10] together with preliminary results. In this article, Set Scheduling is further analyzed and evaluated in a macro-cellular environment in order to fully understand its potential in reducing delay due to resource assignment when compared to Block by Block Scheduling. In order to evaluate Set Scheduling, two different algorithms for organizing RBs in sets are evaluated. Results obtained through the evaluation of Set Scheduling in a macrocellular environment show that it is possible to reduce scheduling delay due to resource assignment by up to four times and to obtain a user capacity improvement of up to $5 \%$ when compared to a Block by Block Scheduling strategy. We will also discuss how it provides a more efficient use of available resources as compared to [8]. Considering that packet delay involves the scheduling process, packet fragmentation and reconstruction (when using CA), signaling and physical layer processes, an improvement in the delay caused by any of these processes will eventually impact packet delay. Therefore, depending on the delay contribution of each process, the achieved reduction in scheduling delay using our proposal may have an important impact in packet delay.

The article is organized as follows: Section 2 presents the scenario and the parameters used for evaluation; Section 3 presents the proposed scheduling strategy, together with the RB organization algorithm; Section 4 shows the delay and throughput analysis for the evaluation scenario and the simulation results; Section 5 presents our conclusions.

\section{Scenario and evaluation parameters}

\subsection{Scenario}

For evaluation, we consider a single cell scenario in a macrocellular urban environment. Figure 1 shows this scenario. Users are uniformly distributed within the base station's (eNodeB) coverage area. Although a uniform user distribution does not fit the general case in practical environments, for a densely populated macrocellular urban scenario it is widely used in literature such as [11]. A uniform user distribution is adequate for algorithm evaluation in this type of scenario when a uniform user density is used.

At each scheduling slot, user requests are received with a Poisson distribution with mean $\lambda_{u}$. Each request 


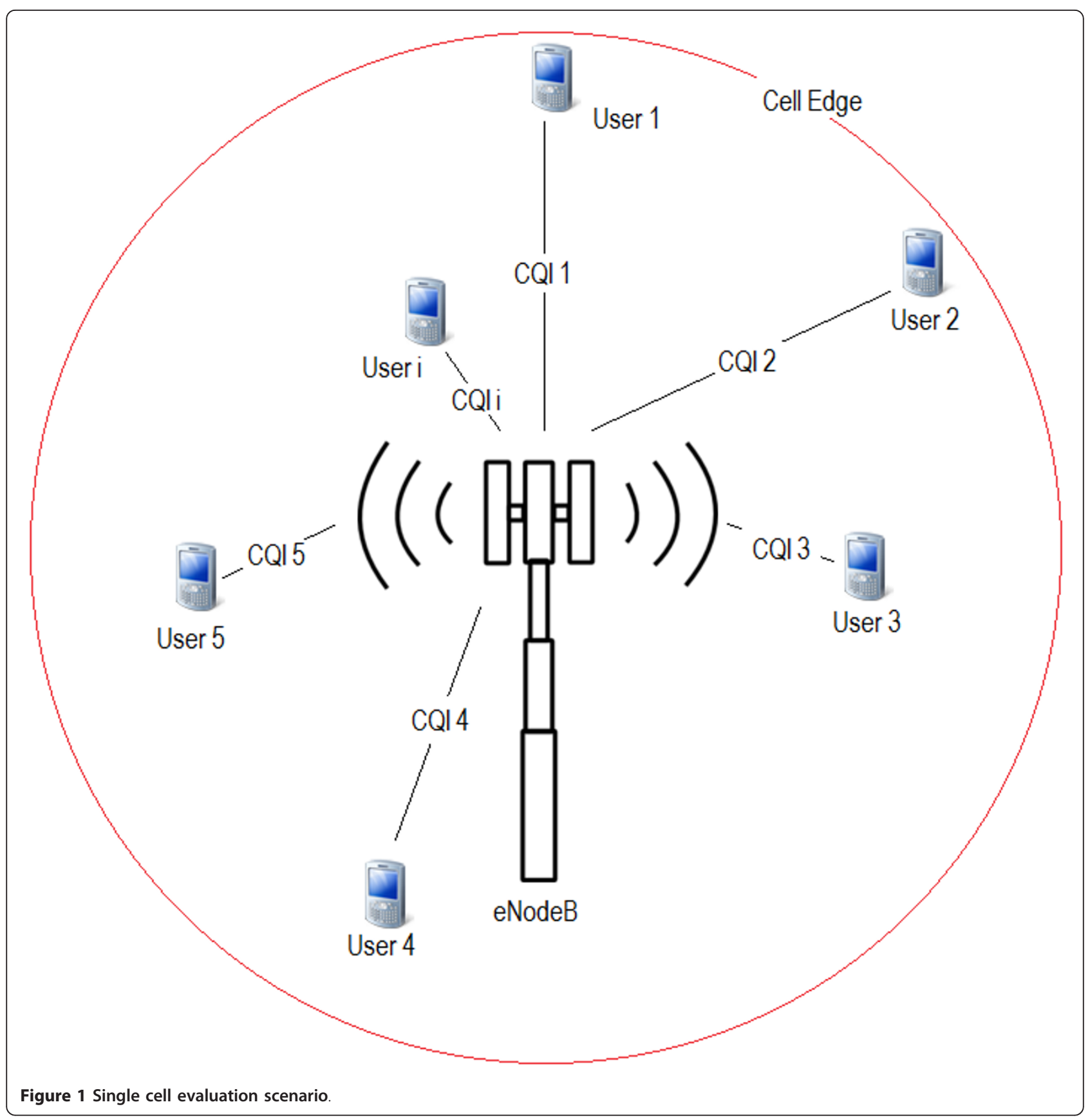

specifies a data rate $R_{b_{u_{i}}}$ and by a file size $S_{b_{u_{i}}}$ for the $i$-th user. Each request is also associated with a channel quality indicator (CQI) report. The CQI will determine the achievable data rate and the amount of data that can be transported from a RB. Therefore the requested data rate will eventually define the bandwidth required by the user (number of required RBs), while the file size will define the number of packets needed to be transmitted (time slots) for a given CQI value.
We consider that the available bandwidth is organized in component carriers (CC) [4]. For evaluation we considered the characteristics of the long term evolution system (LTE) as presented in [12]. There are a total of $\mathrm{L}$ CC's. Each CC $l$, where $l \in 1 \ldots L$, is composed of an integer number RBs, where one RB is the minimum assignable resource to a user. Each RB is by itself a set of OFDM subcarriers. For our evaluation, RBs are represented as binary vectors for each of the frequency bands used. The position within the vector specifies the center 
frequency of the $R B$, a value of " 1 " indicates that the $R B$ is available while a value of " 0 " indicates that the RB is not available.

The achievable data rate per RB is variable, and depends on the CQI of user $i$ on CC $l$. In order to obtain the CQI, the signal to noise ratio (SNR) to CQI mapping presented in [12] was used. This mapping is represented by Equation (1).

$$
\mathrm{CQI}=0.5250 * \mathrm{SNRdB}+4.5 \text {. }
$$

where SNRdB is the user SNR in $\mathrm{dB}$. As presented in [12], this mapping guarantees decodability of the transmitted information with a block error rate (BLER) of at most $10 \%$. Our evaluation considers that requests are from slow moving or fixed users, therefore the CQI is maintained until the transmission of data is completed. For illustration purposes our simulations are based on a single cell multi-user scenario. Interference is not taken into account.

An SNR estimation is required to calculate the CQI. In order to obtain the SNR for each user, we considered the path loss (PL) and thermal noise. The PL was obtained using the ITU-R macrocellular urban NLOS scenario defined in [13] shown in Equation (2).

$$
\begin{aligned}
\mathrm{PL}= & 161.04-7.1 * \log 10(W)+7.5 * \log 10(h) \\
& -\left(24.37-3.7 *\left((h / h b s)^{2}\right)\right) * \log 10(h b s) \\
& +(43.42-3.1 * \log 10(h b s)) *(\log 10(d)-3)(2) \\
& +20 * \log 10(f c) \\
& -\left(3.2 *(\log 10(11.75 * h u t))^{2}-4.97\right) .
\end{aligned}
$$

The parameters for Equation (2) and the values used are taken as the default in [13] and are as follows: PL is the $\mathrm{PL}$ in $\mathrm{dB}, W$ is the street width $(20 \mathrm{~m}), h$ is the average building height $(20 \mathrm{~m}), h b s$ is the base station height $(25 \mathrm{~m})$, hut is the user terminal height $(1.5 \mathrm{~m}), d$ is the distance between user terminal and base station (variable with user position) and $f_{c}$ is the operating frequency in $\mathrm{GHz}$ (2.3 and $3.4 \mathrm{GHz}$ ).

A thermal noise power spectral density of $-174 \mathrm{dBm} /$ $\mathrm{Hz}$ was considered in order to calculate de SNR per user. The $2.3 \mathrm{GHz}$ and $3.4 \mathrm{GHz}$ frequency bands were used with equal transmission power. The transmission power of eNodeB was adjusted in order to have a minimum CQI of 5 at the cell edge in the $2.3 \mathrm{GHz}$ frequency band. Since both bands transmit at the same power, a lower CQI is expected in the $3.4 \mathrm{GHz}$ band for a given user.

The data rates associated to CQI values presented in Table 1 are taken from [14]. In Table 1, S(CQI) represents the transport block size, which defines the amount of data that can be transmitted per RB given a CQI
Table 1 CQI to S(CQI) and R(CQI) mapping

\begin{tabular}{llll}
\hline CQI & Modulation & S(CQI) [bits] & R(CQI) [kbps] \\
\hline CQI 5 & QPSK & 377 & 188.5 \\
CQI 8 & QPSK & 792 & 396.0 \\
CQI 15 & QPSK & 3319 & 1659.5 \\
CQI 22 & 16-QAM & 7168 & 3584.0 \\
\hline
\end{tabular}

value. $R$ (CQI) represents the achievable bit rate for a specific CQI value.

The total number of RBs required by user i, $N_{i}$, is such that:

$$
\sum_{j=1+k_{i-1}}^{N_{i}+k_{i-1}} R(\mathrm{CQI})_{j} \geq R_{b_{u_{i}}} .
$$

where the term $k_{i-1}$ represents the index of the last RB assigned to the previous user.

The restriction in Equation (3) guarantees that the sum rate of the assigned $\mathrm{RBs}$ is at least equal to the data rate required by a user. For a given user $i, N_{i}$ is upper bounded by

$$
N b_{i}^{u}=\frac{R_{b_{u_{i}}}}{R(\mathrm{CQI})_{l}} .
$$

where $N b_{i}^{u}$ is the upper bound on the number of RBs required by user $i$ when all assigned RBs have an equal and the lowest achievable CQI, termed $R(\mathrm{CQI})_{l}$. In the same way, the lower bound on $N_{i}$ can be represented as:

$$
N b_{i}^{l}=\frac{R_{b_{u_{i}}}}{R(\mathrm{CQI})_{h}} .
$$

where $N b_{i}^{l}$ is the lower bound on the number of RBs required by user $i$ when all assigned RBs have an equal and the highest achievable CQI, termed $R(C Q I)_{h}$. Any possible value of $N_{i}$ will fall within these two limits and depends specifically on the user channel conditions on the available carriers.

\subsection{Evaluation parameters}

Table 2 shows the simulation parameters used in all of the evaluations presented in this article, and their corresponding values.

\section{Set Scheduling and resource block organization algorithm}

3.1 Set Scheduling

In order to understand the difference between the proposed Set Scheduling strategy and Block by Block Scheduling, let us first explain the operation of the latter. 
Table 2 Simulation parameters and values

\begin{tabular}{ll}
\hline Parameter & Value \\
\hline Site layout & Single cell, omnidirectional antenna \\
Path loss & ITU-R urban macrocellular NLOS [13] \\
User location & Uniformly dropped within cell \\
Operation frequency & 2.3 and $3.4 \mathrm{GHz}$ \\
Thermal noise PSD & $-174 \mathrm{dBm} / \mathrm{Hz}$ \\
Minimum CQI at cell edge & $5 @ 2.3 \mathrm{GHz}$ \\
Available resource blocks & $20 @ 2.3 \mathrm{GHz}, 160 @ 3.4 \mathrm{GHz}$ \\
Requests per slot $\lambda_{u}$ & 25, Poisson distributed \\
Requested data rate $R_{b_{u_{i}}}$ & Uniformly distributed(1 kbps to $R_{b}$ max) \\
Requested file size $S_{b_{u_{i}}}$ & Uniformly distributed(100 bits to $S_{b}$ max) \\
Simulated slots & 500 \\
\hline
\end{tabular}

Figure 2 shows the general structure of the Block by Block Scheduling strategy presented in [5-7].

In Block by Block Scheduling each available RB is handled individually. Depending on the scheduler used (i.e., Proportional Fair, Processor Sharing) the scheduling metrics are evaluated for each RB. The user who maximizes the specific scheduling metric obtains the $\mathrm{RB}$ assignment. This process is repeated until all RBs are assigned, time at which some users will complete all of their $N_{i}$ required RBs (with $N_{i}$ subject to the constraint in Equation (3)).

We consider that there is an important drawback in a block by block scheduling strategy. For a user $i$ who requires $N_{i}$ RBs, the time required to assign all of them is of at least $N_{i}$ times of that required to assign a single RB. This time can grow even more if the RBs assigned are not contiguous. There is also the possibility that after all the available RBs are assigned, some users will not complete the total number of required RBs. This may result in inefficient use of the available resources.

As a solution to the potentially excessive delay in block by block scheduling, we propose the use a Set Scheduling strategy as shown in Figure 3. Using Set Scheduling, available RBs are first organized in sets prior to assignment by the scheduler. Each set as a whole is available to the scheduler. For a user who requires a total of $N_{i}$ RBs, if a set of size $N_{i}$ or larger is available, it is assigned to that user in a single operation.

There is one main drawback in the proposed Set Scheduling strategy. Additional complexity at the scheduler is required due to the RB organization algorithm. However, depending on the algorithm itself, this complexity can be low compared to the rest of the scheduler components.

\subsection{Resource block organization algorithm}

The operation of the Set Scheduling strategy is subject to the use of a resource block organization algorithm. Figure 4 shows a block diagram of one of the proposed resource block organization algorithms as presented in [10]. The algorithm in Figure 4 will be referred to as Algorithm 1. The operation of Algorithm 1 is based on the search of consecutive available RBs. In this regard,

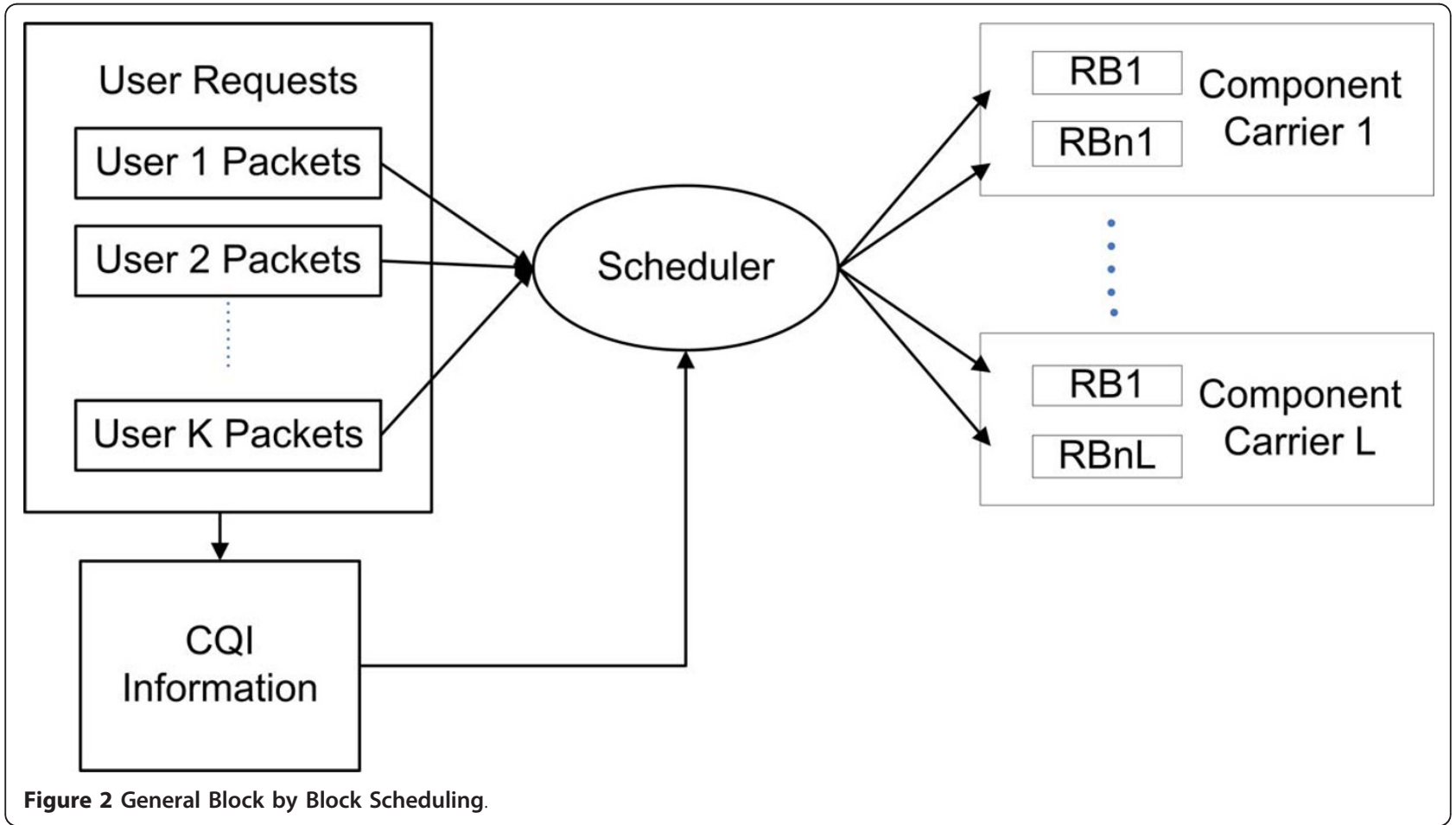




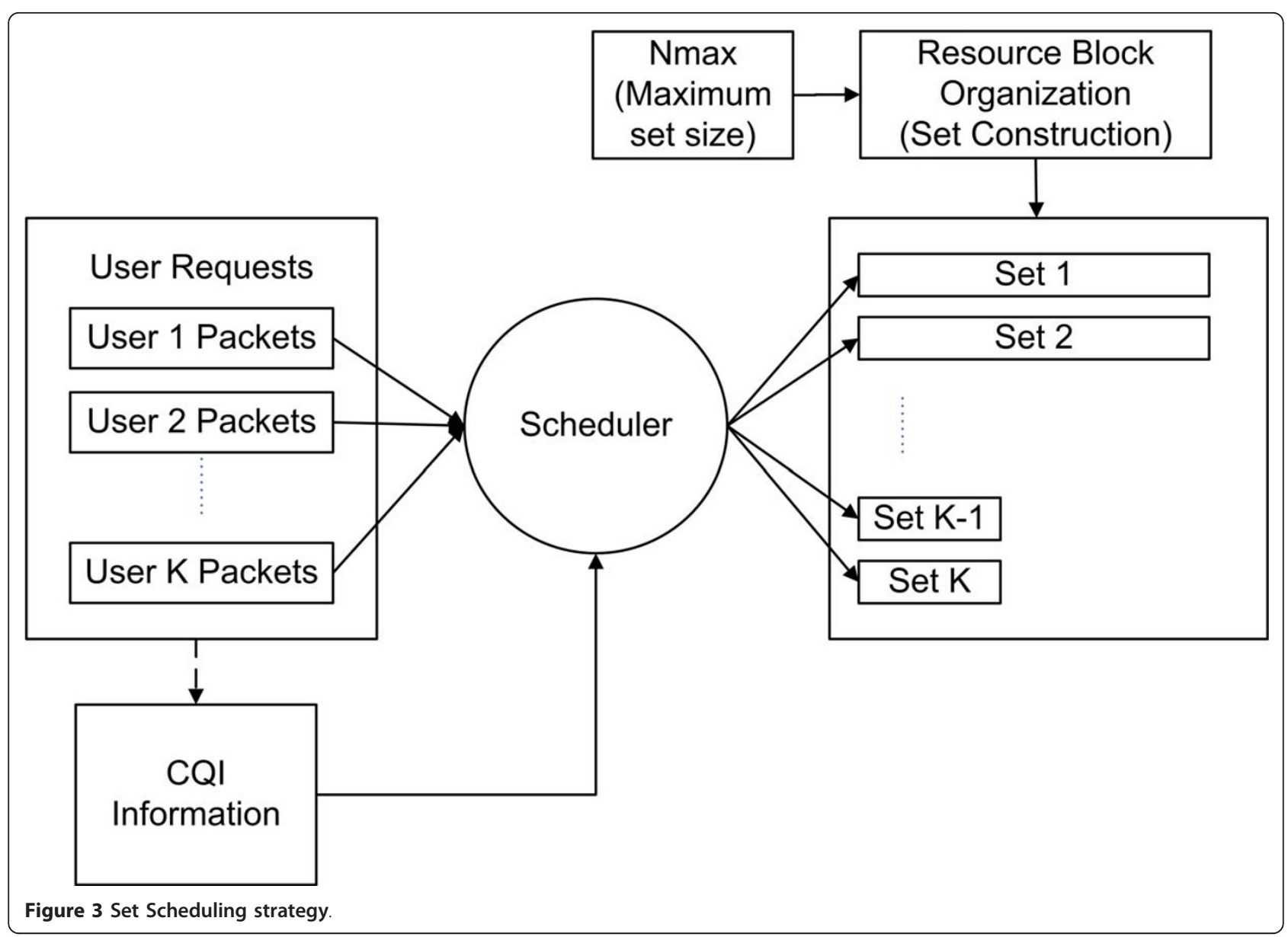

Algorithm 1 finds the first available RB from the vector containing RB availability information, and then checks for the contiguous RB for availability. This process is continued until the contiguous RB checked is not available, or until a total of Nmax contiguous RBs are found.
Nmax specifies the maximum size of a set. The index (position) of each RB found available is stored in a row of a Set Matrix. The last column of the row contains the size of the corresponding set. After a set has been formed, a new set is started by finding the next available

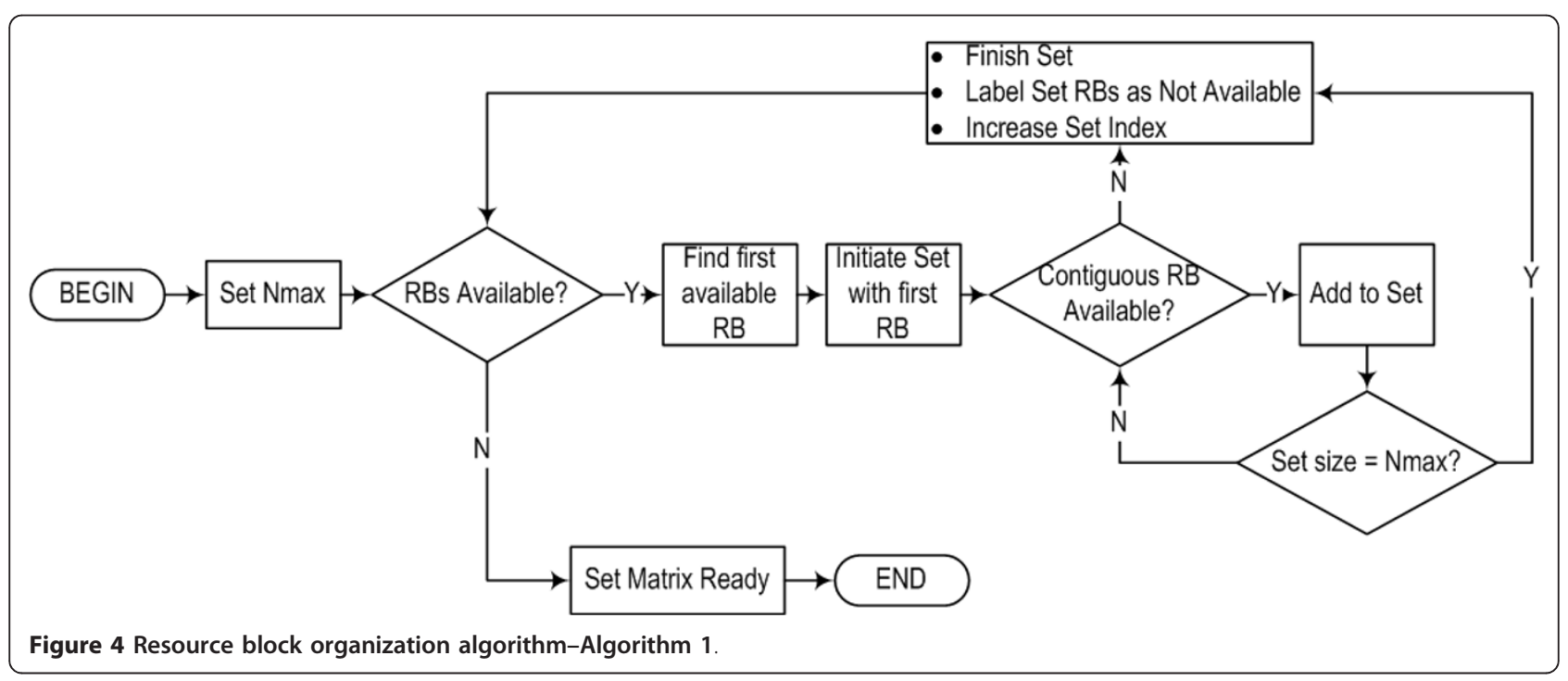


$\mathrm{RB}$, starting the search at the index position where the previous set was finished. The Set Matrix is ready when no more RBs are available to form a set. This process is executed at each scheduling slot, before the assignment of resources.

A different RB Organization algorithm is shown in Figure 5, and will be referred to as Algorithm 2. In Algorithm 2, the output is the same as in Algorithm 1 and corresponds to the Set Matrix. However, the operation is different. At the beginning, Algorithm 2 finds the first available $\mathrm{RB}$ to form a set. The index corresponding to this $\mathrm{RB}$ is considered as the first in the set. Then, Algorithm 2 finds the first non-available RB starting the search at the index of the RB of the previous step. This process can be thought of as a search of the beginning and end of a set. The size of the set is verified, and if it is greater than Nmax then the set found is truncated and a new set is formed immediately with the remaining RBs.

Note that the presented algorithms perform Contiguous CA. This simplifies the organization algorithm operation, but lacks the capacity to form sets from noncontiguous RBs. It has to be remarked that the organization operation is based solely on RB availability.

\subsection{Operation of Set Scheduling}

Algorithms 1 and 2 were used for set construction in the Set Scheduler structure presented in Figure 3. At each scheduling slot, sets are formed using all the available RBs. The scheduler then assigns each available set to users according to the scheduling rules. Due to the dynamic nature of the resource use, each set can have a different size with a minimum size of one, and a maximum size of Nmax.
Once the Set Matrix is ready, the scheduler proceeds to the resource assignment operation. An important restriction of our Set Scheduling evaluation is that sets are assigned to a user if and only if the user has the same CQI for all the RBs in the set. This yields a disadvantage in terms of resource assignment, but it reduces the delay involved in evaluating the constraint of Equation (3). It also guarantees decodability considering that the CQI is adequate for all the RBs in the set. In order to understand the impact of this restriction, consider a user that has different CQI levels in contiguous frequencies that span an available set. For such user, service would be denied until a set that falls within a range of frequencies that have the same CQI level is available. In the best case this restriction results in delayed attention, and in the worst case it would result in an unattended user request. Although this restriction seems to severely affect QoS, in our evaluations with no interference the probability that a user shows different CQI levels within a frequency band $(2.3$ or $3.4 \mathrm{GHz}$ ) is less than $3 \%$. In real world applications, the use of adequate interference control mechanisms reduces the probability that a user experiences different CQI levels within a frequency band. This situation does not occur with Block by Block Scheduling since each RB is handled individually.

In our implementation, during the assignment process the scheduler first looks for a set that matches the number of required RBs for a given user. If such set is not available the scheduler is able to assign a set with a larger number of RBs. Only the required RBs will be assigned. The unused RBs from that set will be used to form a new set. The scheduling slot ends when all sets are assigned or all user requests are attended. Non attended users are queued for the next scheduling slot.

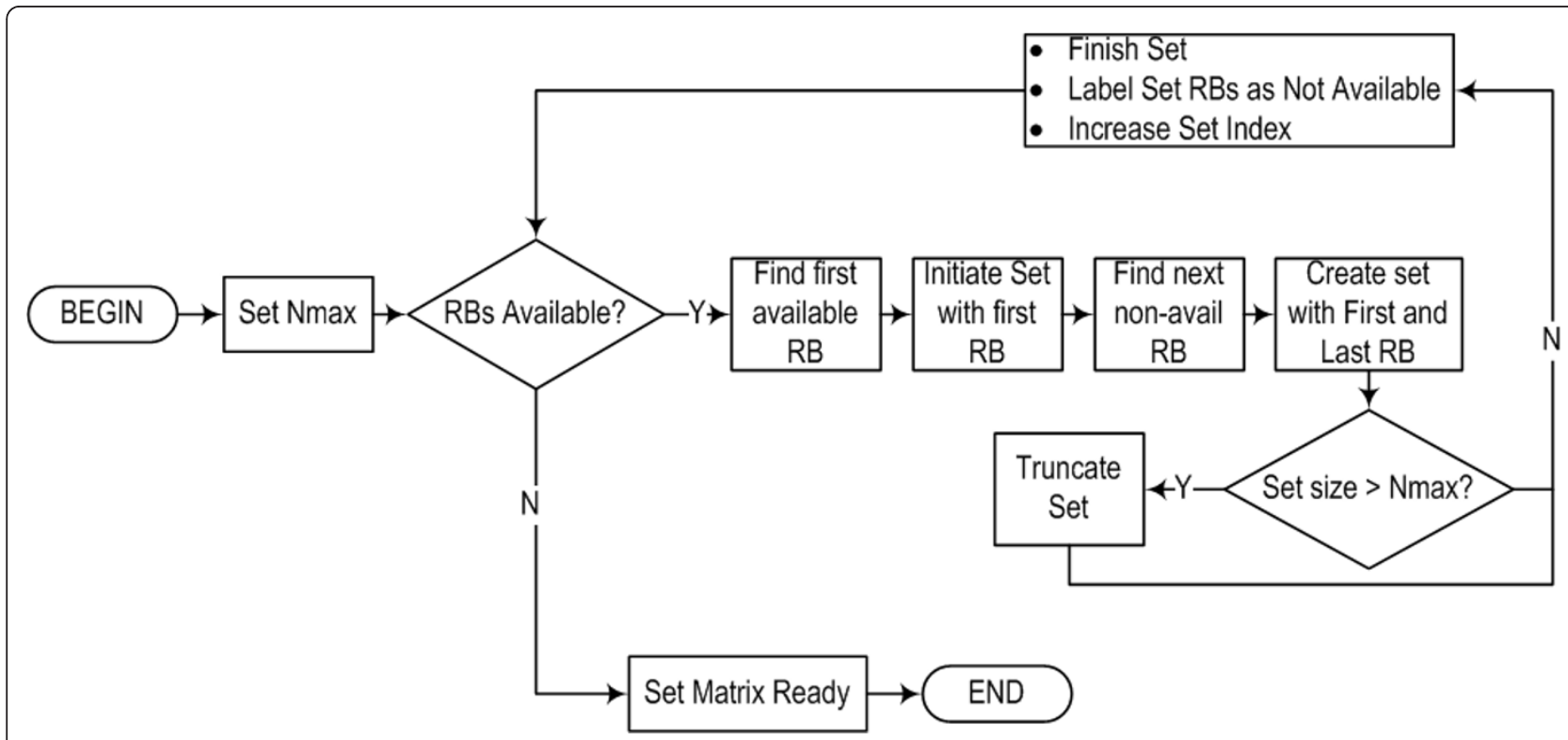

Figure 5 Resource block organization algorithm-Algorithm 2. 
When compared to the scheduling presented in [8], there is an important advantage of using Set Scheduling. In [8] resources are assigned also as sets, but each set corresponds to a complete CC. This means that if a CC is composed of $100 \mathrm{RBs}$ and a user requires $101 \mathrm{RBs}$, a total of $200 \mathrm{RBs}$ will be assigned, corresponding to two CCs. Set Scheduling will only assign as many RBs as required, allowing to use unassigned RBs at a next scheduling slot. Still, it is not possible to directly compare our proposal with that in [8].

\section{Evaluation results and analysis}

Using the parameters from Section 2, numerical evaluation was performed to asses the performance of Set Scheduling in comparison to Block by Block Scheduling. The scheduling strategy in Figure 2 was implemented considering the possibility of non-contiguous inter-band CA subject to the restriction of Equation 3. The scheduling strategy in Figure 3 was implemented as described in Section 3. For evaluation a Round Robin scheduler was used. Although the evaluation scenario is simple, it allows to focus in the assessment of the capabilities of Set Scheduling.

The value of Nmax was evaluated at 15, 18, 20 and 22 RBs. The value of $R_{b}$ max was evaluated between 2,200 and 7,000 kbps and for a given CQI value it determines the average number of $\mathrm{RBs}$ that will be required per user. The value of $S_{b} \max$ was evaluated at 2000, 3000, 4000 and 5000 bits, and for a given CQI value it determines the average number of time slots required to complete a user request transmission.

\subsection{Delay analysis}

The expected delay due to resource assignment in block by block scheduling can be estimated using Equation 6 .

$$
E[\text { Delay }]=E[N] \cdot \tau_{s} .
$$

where $E[$ Delay] is the expected delay to assign all the required RBs to a given user, $E[N]$ is the expected number of RBs per user and $\tau_{s}$ is the time required to assign one single $\mathrm{RB}$.

To calculate $E[N]$ we will use the conditional expectation method. Thus note that for a given CQI value $N$ can be calculated as:

$$
\begin{aligned}
& N=\frac{R_{b_{u}}}{R(\mathrm{CQI})} \\
& \forall R(\mathrm{CQI})>0
\end{aligned}
$$

where $R_{b_{u}}$ is a random variable and $\mathrm{R}(\mathrm{CQI})$ is assumed to be constant and non-zero. There-fore we can obtain

$$
\begin{gathered}
E[N]=E\left[\frac{R_{b_{u}}}{R(\mathrm{CQI})}\right]=\frac{1}{R(\mathrm{CQI})} E\left[R_{b_{u}}\right] \\
\forall R(\mathrm{CQI})>0,
\end{gathered}
$$

Now, in order to obtain $E[N]$, we use

$$
\begin{aligned}
E[N]= & E[E[N \mid R(\mathrm{CQI})]]=E\left[\frac{1}{R(\mathrm{CQI})} E\left[R_{b_{u}}\right]\right] \\
& \forall R(\mathrm{CQI})>0,
\end{aligned}
$$

Given the restriction of $R(\mathrm{CQI})>0$ and the statistical independence between $R(\mathrm{CQI})$ and $R_{b_{u}}$, we can now write

$$
E[N]=E\left[\frac{1}{R(\mathrm{CQI})} \mid R(\mathrm{CQI})>0\right] E\left[R_{b_{u}}\right] .
$$

where considering $\mathrm{R}(\mathrm{CQI})$ as a discrete random variable with possible values indicated in Table 1 , the term $E\left[\frac{1}{R(\mathrm{CQI})} \mid R(\mathrm{CQI})>0\right]$ can be obtained using the following

$$
E\left[\frac{1}{R(\mathrm{CQI})} \mid R(\mathrm{CQI})>0\right]=\sum \frac{1}{R(\mathrm{CQI})} p(R(\mathrm{CQI}) \mid R(\mathrm{CQI})>0) .
$$

Note that $\mathrm{R}(\mathrm{CQI})$ depends directly on the CQI value. A numerical analysis of the CQI for the evaluation scenario and parameters of Section 2, showed that the CQI behaves as a random process with exponential probability density function (pdf). This behavior was verified using $Q-Q$ plots showing a perfect fit. Considering this and the discretization of $\mathrm{R}$ (CQI) due to the mapping in Table 1, it is possible to obtain specific values for the term $E\left[\frac{1}{R(\mathrm{CQI})} \mid R(\mathrm{CQI})>0\right]$. Numerical evaluation found that for the $2.3 \mathrm{GHz}$ band there is an $E[\mathrm{CQI}]=$ 8.85, while for the $3.4 \mathrm{GHz}$ band $E[\mathrm{CQI}]=7.12$. For the $2.3 \mathrm{GHz}$ frequency band,

$$
E\left[\frac{1}{R(\mathrm{CQI})} \mid R(\mathrm{CQI})>0\right]=0.00251=\frac{1}{399 \mathrm{kbps}} .
$$

Since this value corresponds to a data rate per RB not supported, it possible to say that the value would be in fact $\frac{1}{396 \mathrm{kbps}}$ for the $2.3 \mathrm{GHz}$ frequency band. In the same fashion, it was found that for the $3.4 \mathrm{GHz}$ frequency band

$$
E\left[\frac{1}{R(\mathrm{CQI})} \mid R(\mathrm{CQI})>0\right]=0.00292=\frac{1}{343 \mathrm{kbps}} .
$$


which given the discretization of $\mathrm{R}(\mathrm{CQI})$ results in a value of $\frac{1}{188.5 \mathrm{kbps}}$.

Given the uniform distribution of the data rate requested described in Section 2, we can assume that $E\left[R_{b_{u}}\right]=R_{b} \max / 2$. Once the value of $E[N]$ is obtained using (10), the expected delay for the assignment of resources in Block by Block Scheduling in terms of $\tau_{s}$ can be calculated using Equation (6).

The expected delay when using Set Scheduling can be estimated using Equation (14).

$$
E\left[\text { Delay }_{\text {set }}\right]=E\left[\tau_{o}\right] / \lambda_{u}+\tau_{s} .
$$

where $E\left[\right.$ Delay $\left._{\text {set }}\right]$ represents the expected delay per user due to resource assignment using Set Scheduling; $E$ $\left[\tau_{o}\right]$ represents the time required by the RB organization algorithm to obtain the Set Matrix; $\lambda_{u}$ is the average number of user requests per scheduling slot.

Using numeric evaluation, both RB organization algorithms were evaluated in order to determine the parameter $E\left[\tau_{o}\right]$ for the evaluation conditions specified in Section 2. Figure 6 shows the evaluation of $E\left[\tau_{o}\right]$ for the minimum and the maximum values of $N$ max, with respect to the percentage of available RBs. For this evaluation, available RBs were randomly distributed. The parameter $E\left[\tau_{o}\right]$ is expressed in terms of $\tau_{s}$.

For Algorithm 1 the maximum value of $E\left[\tau_{o}\right]$ is obtained when all RBs are available, and in the worst case for a value of $\mathrm{Nmax}=22$, it corresponds to $49 \cdot \tau_{s}$. Using Equation (14), for the preceded worst case scenario, the expected delay due to resource assignment using Set Scheduling corresponds to $E\left[\right.$ Delay $\left._{\text {set }}\right]=49$. $\tau_{s} / 25+\tau_{s}=2.96 \cdot \tau_{s}$. This delay calculation involves only

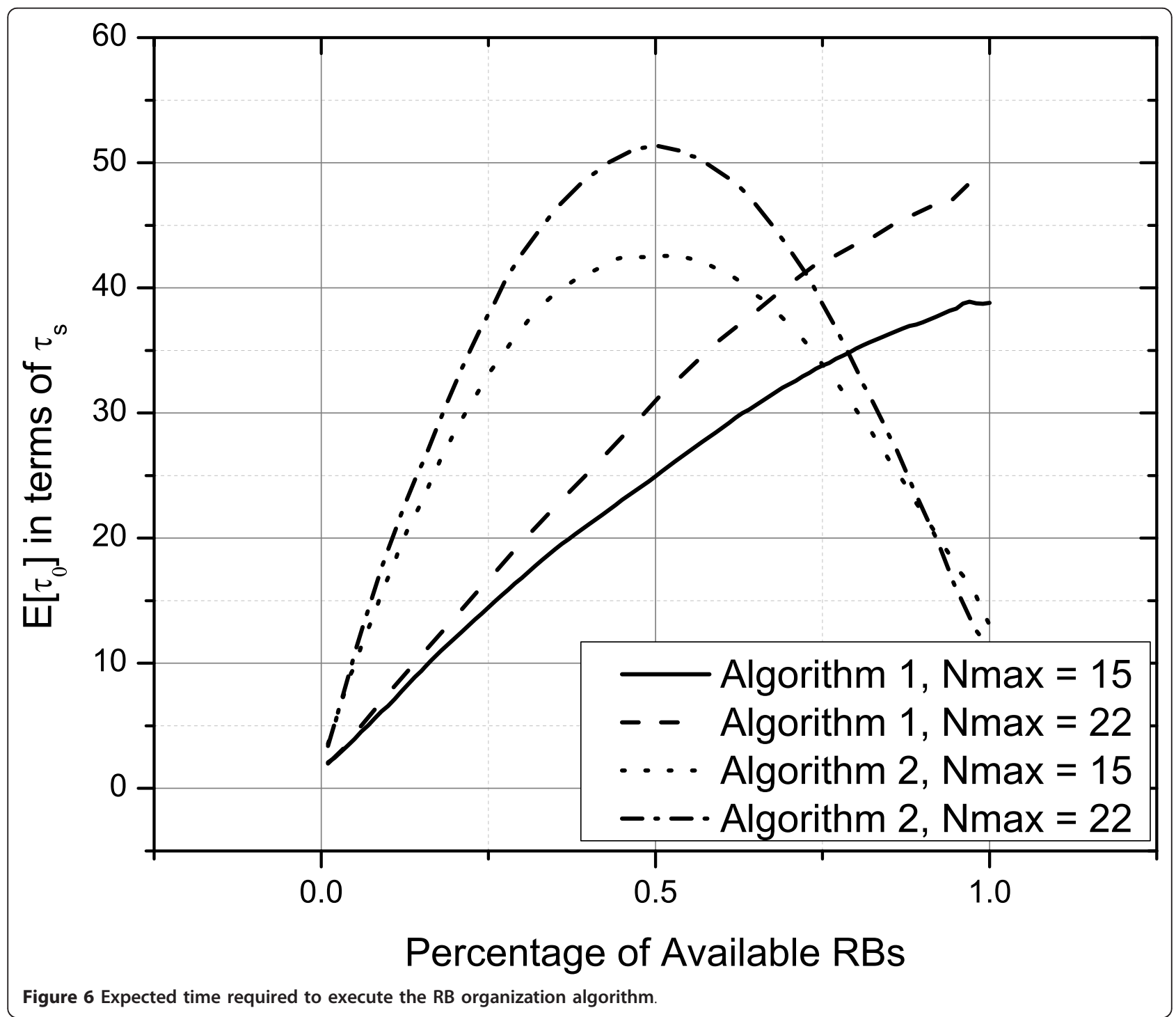


the availability of RBs and the value of Nmax. For this calculation, it is considered that all user requests are attended.

As it can be observed in Figure 6 there is an important difference in the behavior of the proposed algorithms. Algorithm 1 has a monotonically increasing response with respect to available RBs. Algorithm 2 has a parabolic behavior with its maximum at the point where $50 \%$ of RBs are available. When the percentage of RBs is below 70\%, Algorithm 1 outperforms Algorithm 2 in terms of $E\left[\tau_{o}\right]$. However, when a higher percentage of RBs is available for scheduling Algorithm 2 shows a much lower delay. This translates in the fact that when resources are more fragmented, Algorithm 1 will show a lower delay than Algorithm 2. This information is valuable since it makes possible to select an algorithm based on the expected availability of RBs. It is possible to have both algorithms in a system and switch between them depending on the resource availability in order to reduce resource assignment delay.

It is also possible to observe in Figure 6 that the expected delay $E\left[\tau_{o}\right]$ is also dependant on the value of Nmax. For a larger value of Nmax a higher $E\left[\tau_{o}\right]$ can be expected. In Algorithm 1, the worst case of delay shows that for $\operatorname{Nmax}=22, E\left[\tau_{o}\right]=49$, while for $\mathrm{Nmax}=15, E$ $\left[\tau_{o}\right]=38$. This is a significative difference that can also be observed for Algorithm 2. Given this behavior, in order to reduce delay as much as possible the lowest possible value of Nmax has to be selected.

Figure 7 shows a comparison between the expected delay of Block by Block Scheduling for the different values of $E\left[R_{b_{u}}\right]$ and the two frequency bands, with the expected delay for resource assignment when using Set Scheduling in the worst case of Algorithm 1. The delay advantage is obvious. When using Set Scheduling, delay is not dependent of $E\left[R_{b_{u}}\right]$, but rather on the

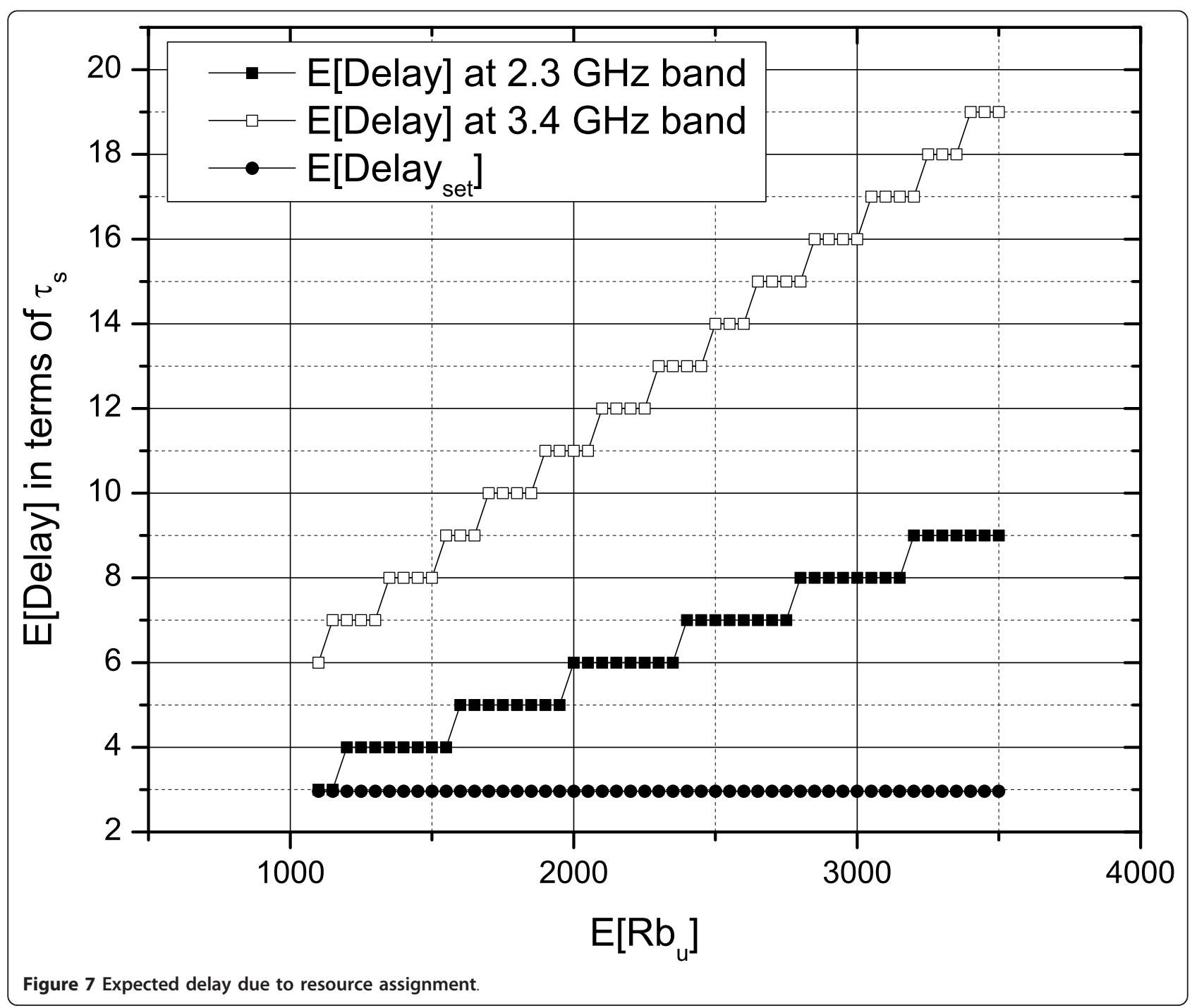


parameters of the $\mathrm{RB}$ organization algorithm and $\mathrm{RB}$ availability. It is also independent on the frequency band. The delay in block by block scheduling is dependent on both $E\left[R_{b_{u}}\right]$ and the frequency band, given that the operating frequency determines the number of expected RBs required per user. For the evaluation parameters used, Set Scheduling takes at most the same delay as Block by Block Scheduling for resource assignment. When compared to the $3.4 \mathrm{GHz}$ band it can reduce the delay by up to six times.

\subsection{Complexity description}

In order to compare the complexity of Block by Block Scheduling and Set Scheduling we present the general operation of both strategies. Only the general case for each process is described for comparison. The operations not included in each process are the same for each strategy, and involve frequency band distinction and restrictions such as the maximum value of RBs per user (Nmax).

Procedure 1 shows the general operation of Block by Block Scheduling. For each user request, this strategy will find and assign as many RBs as required in order to meet the restriction in Equation 3. Therefore, for a total of $N_{i}$ RBs, each user needs a total of $N_{i}$ find operations, as well as $N_{i}$ assign and $N_{i}$ update operations. As previously discussed, the delay due to resource assignment using this strategy will in fact depend on the value of $N_{i}$. Since LTE-Advanced systems allow up to $500 \mathrm{RBs}$ to be assigned to a single user in order to exceed the 1 Gbps requirement for IMT-Advance systems, the delay of Block by Block Scheduling can become considerably high. However, it has the advantage that each available RB can be optimally used for a given CQI value. The achievable data rate will be considered independently for each assigned RB.

Procedure 2 shows the general operation of Set Scheduling. In this procedure, each user request within the assignment process requires only one calculate, one find operation and one assign operation. Since the number of required RBs is known due to the restriction of equal CQI for the RBs in a set, no update operation is required. In Set Scheduling, the main cause of delay is the execution of the resource block organization algorithm at each scheduling slot. However, as it was presented in Section 4.1, the organization of available RBs in sets depends mainly on the availability of RBs and the implementation of the organization algorithm. Since the $\mathrm{RB}$ organization algorithm is executed once per scheduling slot, the delay due to its execution can be considered as "distributed" among the attended users.

From the algorithms presented in Section 4.1, a complexity comparison between both strategies is possible. Consider the construction of one set and its assignment to one user. Table 3 shows a comparison in terms of the number of operations that each strategy performs in order to assign the required RBs to a given user. Each operation is considered as having the same complexity. Although not all the operations are shown, Table 3 does allow for a general comparison. In general, a total of $3 N_{i}$ operations are required by Block by Block Scheduling in order to assign a total of $N_{i}$ RBs to a user. On the other hand, Set Scheduling requires a total of $N_{i}+4$ operations when using the RB Organization Algorithm 1, and a total of five operations when using the RB Organization Algorithm 2. We observed that the most time consuming operation within our simulation environment is the find operation. The check operation corresponds to the verification of contiguous RBs in Algorithm 1 (see Figure 4). Therefore, the main difference between both algorithms is that Algorithm 1 uses one find operation and $N_{i}$ check operations per set, while Algorithm 2 performs two find operations per set. For any case, the number of operations performed by Set Scheduling including the RB organization algorithm is lower than for Block by Block Scheduling, with an exception when the number of required RBs is $N_{i}=1$. The complexity advantage of Set Scheduling increases with $N_{i}$.

\subsection{User capacity analysis}

In order to evaluate the performance of Set Scheduling in terms of user capacity, we derived a metric that represents the percentage of user requests that remain in the scheduler queue after a given number of user

Table 3 Comparison of the number of operations required per attended user considering Block by Block Scheduling and Set Scheduling

\begin{tabular}{lccc}
\hline Operation & \multicolumn{3}{c}{ Number of operations } \\
\cline { 2 - 4 } & B by B scheduling & Set Scheduling & RB org. Algorithm. 1, (Algorithm. 2) \\
\hline Find & $N_{i}$ & 1 & $1,(2)$ \\
Assign & $N_{i}$ & 1 & $0,(0)$ \\
Update & $N_{i}$ & 0 & $0,(0)$ \\
Calculate & 0 & 1 & $0,(0)$ \\
Check & 0 & 0 & $N_{i t}(0)$ \\
Total & $3 N_{i}$ & & $N_{i}+4,(5)$ \\
\hline
\end{tabular}


drops. The number of user drops used in our evaluation corresponds to 500 as presented in Section 2. This amount of user drops was obtained through a generate and test algorithm, given that simulating a larger number of user drops does not change the user capacity metric. Equation (15) shows how the metric is calculated

$$
\mathrm{PQ}=1-\left(U_{\mathrm{att}} / U_{\mathrm{rec}}\right)
$$

where PQ is the percentage of user requests in queue; $U_{\text {att }}$ represents the number of attended requests; $U_{\text {rec }}$ corresponds to the number of received requests.

Figure 8 shows a comparison of the PQ metric between Block by Block Scheduling and Set Scheduling at $S_{b} \max =2,000$ bit. The lower value is better. Although the different Set Scheduling evaluations vary in performance, there is always one that outperforms the Block by Block Scheduling behavior. For an $S_{b} \max =$ 2,000 bit the best performance is obtained when Nmax $=20$, with a PQ metric up to $5 \%$ lower than that of Block by Block scheduling (achieved at $R_{b} \max =5,800$ kbps).

Figure 9 shows the same comparison but with $S_{b} \max$ $=5,000$ bit. As it can be observed, the best performance in terms of the PQ metric is obtained with Set Scheduling with $\mathrm{Nmax}=18$. It is possible to note that the value of Nmax that minimizes the PQ metric varies depending on traffic demands. This brings the opportunity to use statistical traffic information in order to select the best possible value of Nmax at each scheduling slot in an adaptive form.

For Figures 8 and 9 it is also possible to bring the information provided by the Set Scheduling delay analysis. Using either one of the proposed algorithms for set

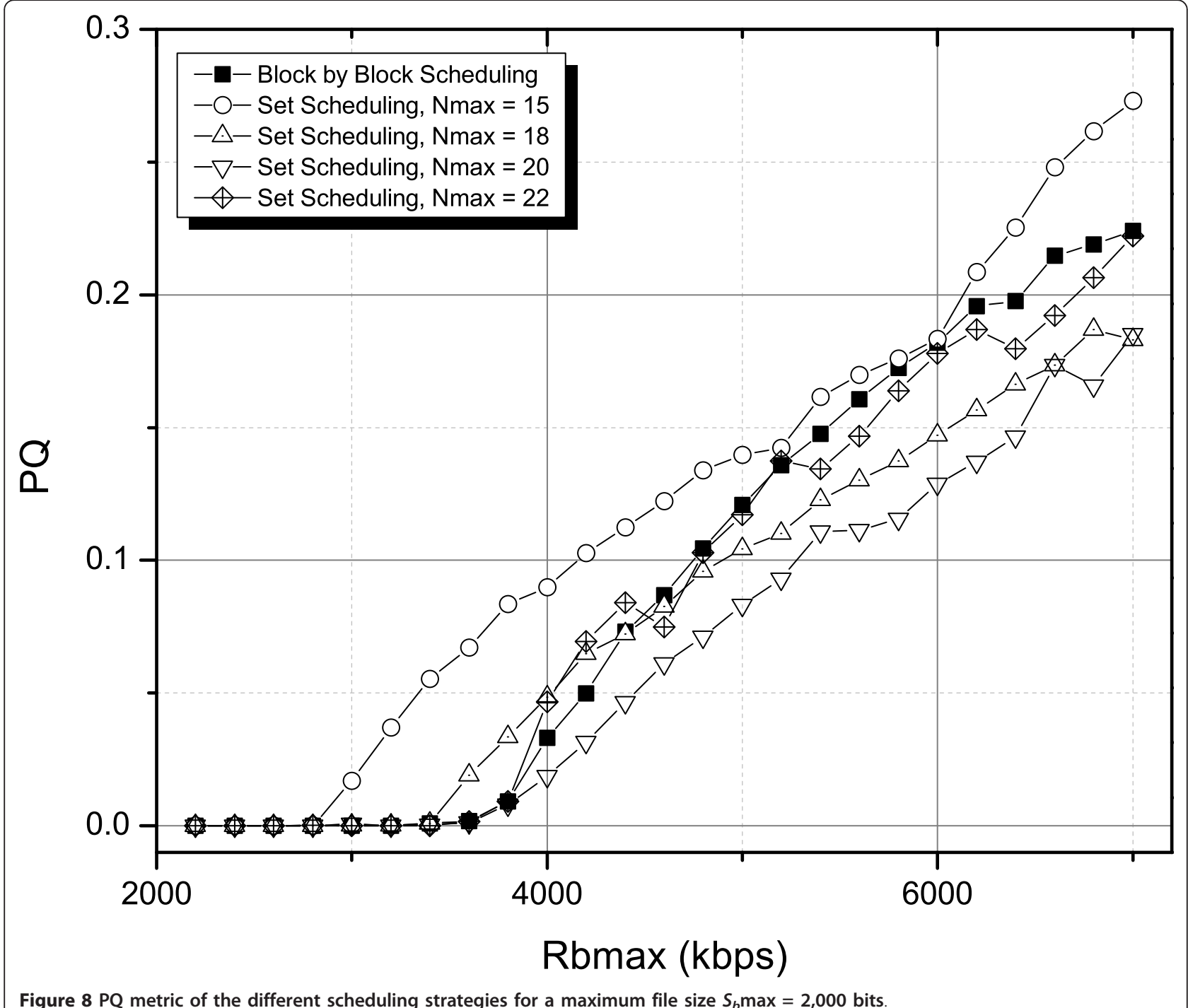




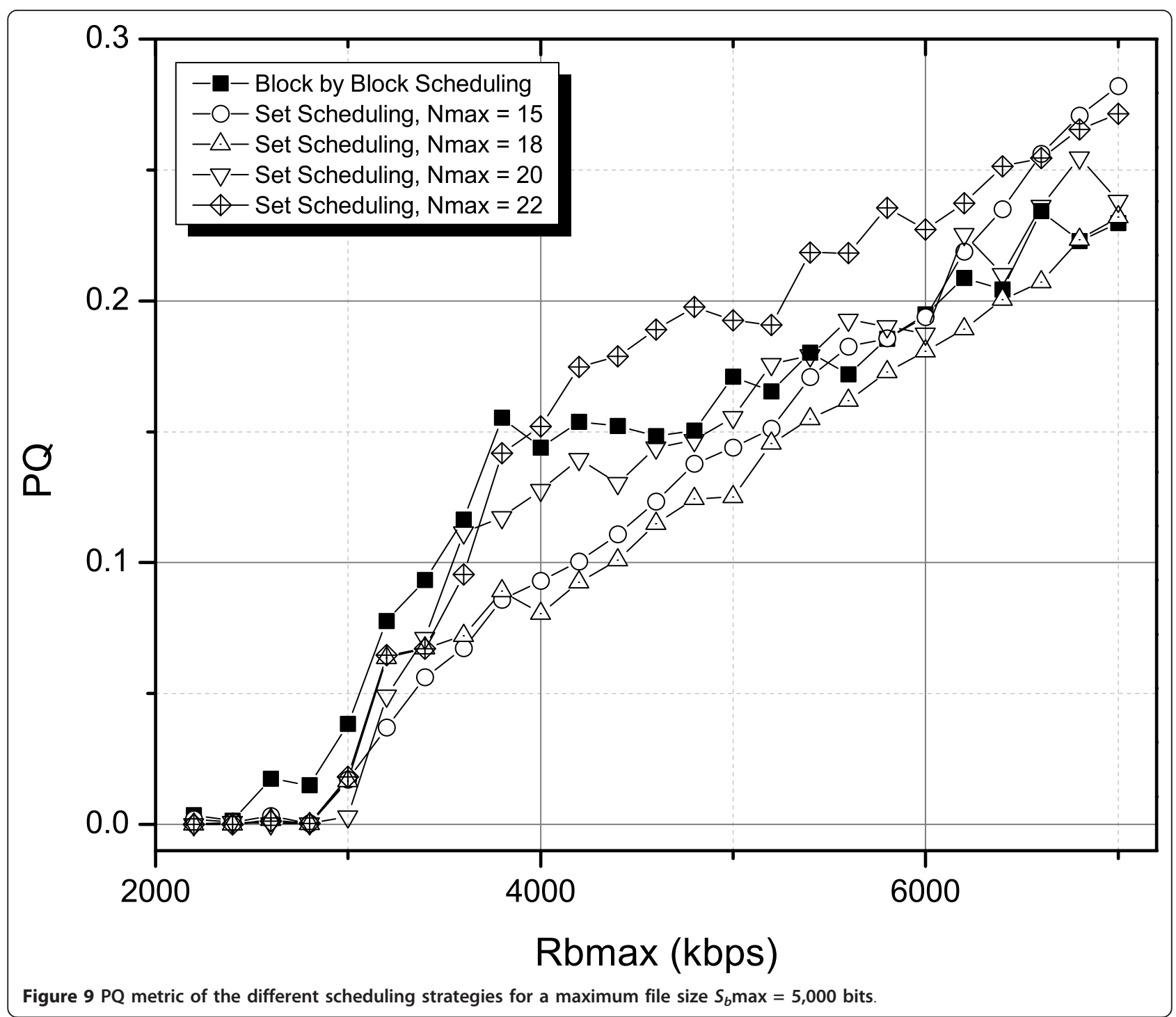

construction, the lowest possible delay is achieved with the lowest value of Nmax. Therefore, from the PQ metric analysis, when two or more performance curves overlap the best selection of Nmax will correspond to the lowest value. As such, in Figure 8 a value of Nmax $=15$ will be preferred for values of $R_{b}$ max lower than $3,000 \mathrm{kbps}$, while for values of $R_{b}$ max between 3,000 and 3,500 kbps a value of Nmax $=18$ is preferred. For $R_{b}$ max greater than $4,000 \mathrm{kbps}$ a value of $\mathrm{Nmax}=20$ performs the best.

Figure 10 compares the best performing results for the different values of $S_{b} \max$ that were evaluated. It is important to note that, for the evaluated conditions, for larger file sizes a smaller value of Nmax performed better. As expected, at higher $S_{b}$ max the PQ metric is increased. In all of the evaluations, Block by Block
Scheduling was outperformed by at least one Set Scheduling configuration.

The strong fluctuations observed in Figures 8, 9 and 10 occur due to the reduction of available resources and the randomness of the simulations performed. Once the average requested data rate increases to a point where it is not possible to attend all requests at every scheduling slot, the PQ metric starts to increase indicating a reduction in system capacity. Also, it can be observed that the average data rate at which the system cannot attend all requests is lower as the average user file size increases. This is particularly visible in Figure 10, where all curves show a very similar slope that starts to increase at a lower value of parameter $R_{b}$ max as $S_{b}$ max increases. This behavior clearly represents the fact that as $S_{b} \max$ increases, fewer RBs are available at each scheduling 


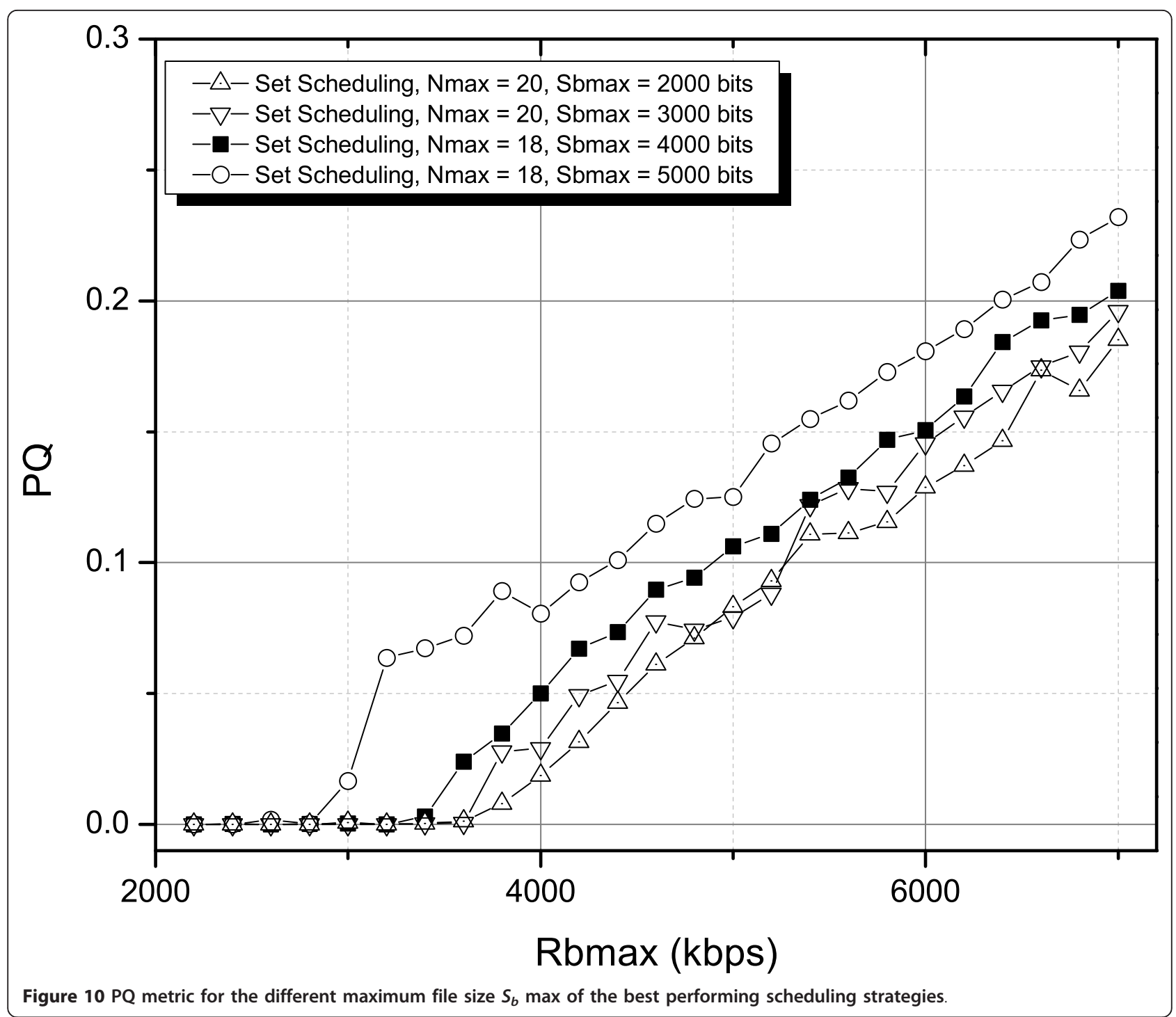

slot, reaching the point of system resource depletion at a lower value of average user requested data rate.

The lower PQ metric means that the cell capacity is increased. Statistically, overall throughput can be calculated by multiplying the PQ metric times the mean data rate requested and the average number of users. Once the PQ metric is greater than zero, all of the available RBs are used at each scheduling slot, indicating that the throughput is at a maximum possible for the scheduling and traffic conditions.

\subsection{Throughput evaluation}

Since the PQ metric used to evaluate user capacity is not commonly used in literature, in this section we provide an evaluation of the throughput behavior of the proposed Set Scheduling strategy. The simulation parameters used to evaluate throughput are shown in Table
2 . The maximum requested bit rate $R_{b}$ max was evaluated from 2,000 to $10,000 \mathrm{kbps}$, and $S_{b}$ max was evaluated for 2,000 and 6,000 bits. For Set Scheduling, a value of $\mathrm{Nmax}=20$ was used.

Figure 11 shows the throughput percentage calculated using Equation 16.

Throughput percentage $=$ Total assigned throughput/Total requested throughput.

Equation 16 allows to compare Block by Block Scheduling and Set Scheduling fairly. From Figure 11 it is possible to observe that for both scheduling strategies, for a larger value of $S_{b}$ max the Throughput Percentage decays. This is due to the fact that as the file size increases, the number of time slots required by the user to complete a transfer also increases, thus reducing the number of available RBs at each scheduling slot. It is also possible to observe that in each case of $S_{b}$ max, Set 


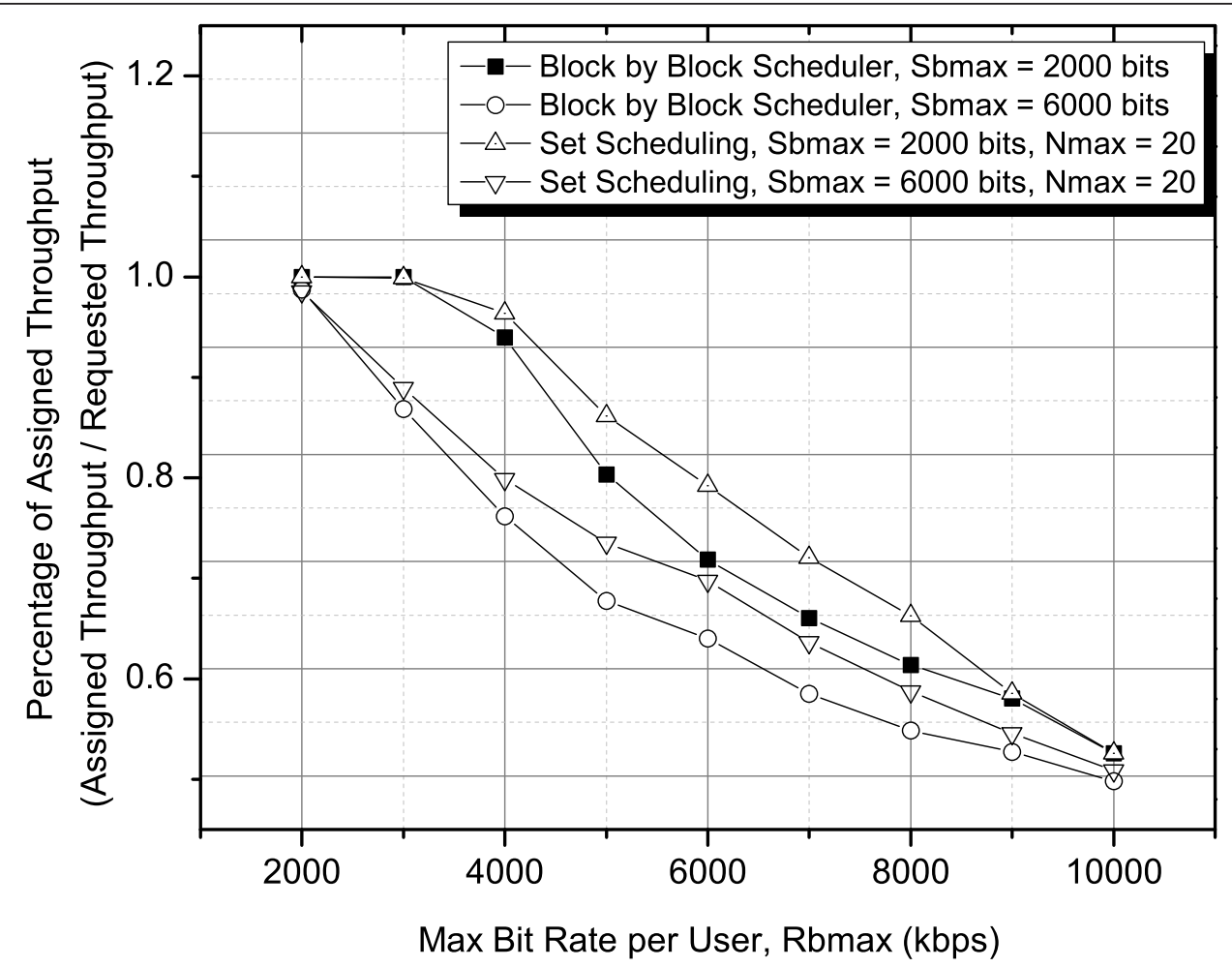

Figure 11 Throughput percentage (assigned throughput/requested throughput) for Block by Block Scheduling and Set Scheduling

Scheduling outperforms Block by Block Scheduling by up to 8 percent observed at a value of $R_{b} \max =6,000$ kbps. However, this advantage is reduced as $R_{b} \max$ increases. This is due to the fact that at some point the maximum throughput that can be handled by the system is reached by both scheduling strategies. This point is reached when $R_{b}$ max is $10,000 \mathrm{kbps}$ for a value of $S_{b}$ $\max =2,000$ bits.

Figure 12 shows the average user throughput assigned by the schedulers. It is possible to observe the saturation of system resources as $R_{b}$ max increases. For a given value of $S_{b} \max$, Set Scheduling outperforms Block by Block Scheduling until the maximum throughput that the system can handle is reached. This behavior is consistent with that in Figure 11.

\section{Conclusions}

A scheduling strategy for CA using pre-organized RB sets was presented and evaluated. We presented an analytical evaluation framework to determine the expected number of RBs required by users, based on a mapping of CQI values to data rates per RB and the statistical behavior of the CQI. This framework allowed us to evaluate a macrocellular environment in order to determine the potential delay advantage of using Set Scheduling.
Two different RB Organization Algorithms were implemented. It was possible to observe a marked difference in the delay behavior of the evaluated algorithms in terms of the percentage of available RBs. A dependance to the percentage of available RBs as well as to the value of Nmax are observed. This opens the possibility of designing a different $\mathrm{RB}$ organization algorithm with improved behavior and lower delay when compared to the algorithms presented. The capacity of reducing delay due to resource assignment using Set Scheduling depends directly on the performance of the RB Organization Algorithm.

Although the RB organization algorithm used provided only contiguous CA functionality, it still outperformed a block by block scheduler that used noncontiguous inter-band CA. Some of the improvements that can be made to the scheduling strategy presented in this article include the possibility of aggregating sets. Set aggregation can improve throughput. Also, it is possible to design a different type of scheduler whose metrics are calculated for a whole set. Another improvement is the possibility of implementing an adaptive RB organization algorithm, that takes into account the statistical behavior of user requests.

In general, we were able to show that Set Scheduling has the capacity of reducing the delay due to resource 


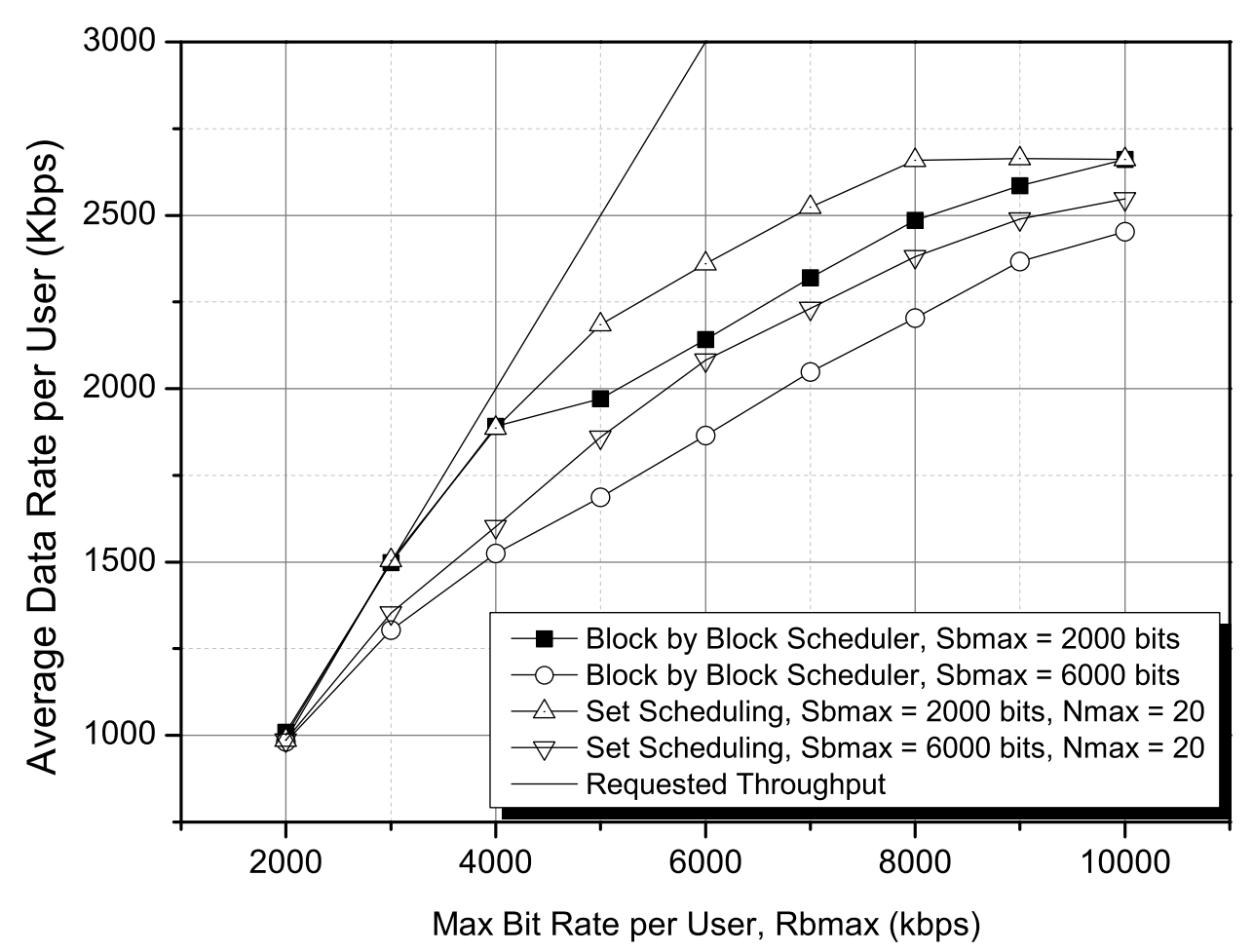

Figure 12 Average assigned throughput per user for Block by Block Scheduling and Set Scheduling.

assignment when compared to Block by Block Scheduling without affecting user capacity measured with the PQ metric, throughput percentage and average user throughput.

\section{Procedure 1 General Block by Block Scheduling process}

$i$ is the index for the user requests

$j$ is the index for the RB vector

$R(\mathrm{CQI})_{j}$ is the achievable data rate for $\mathrm{RB}_{j}$

for Each scheduling slot do

while RBs Available do

if User requests in queue then

$i \leftarrow$ User Index

Updated Sum Rate $\leftarrow$ User $i$ Requested Data

Rate

while Updated Sum Rate $>0$ do

find: Available $R B_{j}$

assign: $R B_{j}$ to User $i$

Rate - $R(\mathrm{CQI})_{j}$

update: Updated Sum Rate $=$ Updated Sum

end while

increment: User Index

else

completed

break: No more user requests, process end if end while

end for

\section{Procedure 2 General Set Scheduling process}

for Each scheduling slot do

execute: Resource Block Organization Algorithm

while RB Set Available do

if User requests in queue then

$i \leftarrow$ User Index

calculate $N_{i} \leftarrow$ Number of required RBs for user $i$ for the different CQI values

find: Set with size $\geq N_{i}$

assign: $N_{i}$ RBs from set to User $i$

increment: User Index

else

break: No more user requests, process

completed

end if

end while

end for

\section{Acknowledgements}

This research work was possible thanks to scholarship number 92845 granted by the National Council of Science and Technology (CONACYT, Mexico) and to the support provided by the Wireless Communications Group at the CICESE Research Center and the Autonomous University of Baja California (UABC), Mexico. 


\section{Author details}

'Department of Electronics and Communications, CICESE Research Center, Ensenada, Mexico ${ }^{2}$ Department of Electrical Engineering, School of Engineering, Autonomous University of Baja California, Mexicali, Mexico

\section{Competing interests}

The authors declare that they have no competing interests.

Received: 15 October 2011 Accepted: 14 March 2012 Published: 14 March 2012

\section{References}

1. M Dötling, W Mohr, A Osserain, Radio Technologies and Concepts for IMTAdvanced, (Wiley, NJ, USA, 2009)

2. E Dahlman, S Parkvall, J Sköld, 4G LTE/LTE-Advanced for Mobile Broadband, (Academic Press, Oxford, UK, 2011)

3. M Lazarus, The great spectrum famine. IEEE Spectr. 47(10), 26-31 (2010)

4. G Yuan, X Zhang, Y Yang, Carrier aggregation for LTE-advanced mobile communication systems. IEEE Commun Mag. 48(2), 88-93 (2010)

5. L Lei, K Zheng, Performance evaluation of carrier aggregation for elastic traffic for LTE-advanced systems. IEICE Trans Commun. E92-B(11), 3516-3519 (2009). doi:10.1587/transcom.E92.B.3516

6. S Songsong, F Chunyan, G Caili, A resource scheduling algorithm based on user grouping for LTE-advanced system with carrier aggregation, in Computer Network and Multimedia Technology, 2009. CNMT 2009. International Symposium, Wuhan, China, (18-20 Dec 2009)

7. L Chen, W Chen, X Zhang, D Yang, Analysis and simulation for spectrum aggregation in LTE-advanced system, in Vehicular Technology Conference Fall (VTC 2009-Fall). 2009 IEEE 70th, Anchorage, Alaska, USA (20-23 Sept 2009)

8. Y Chungm, Z Tsai, A quantized water-filling packet scheduling scheme for downlink transmissions in LTE-Advanced systems with carrier aggregation, in Software, Telecommunications and Computer Networks (SoftCOM), 2010 International Conference on, Split-Adriatic Islands, Croacia, pp. 275-279 (2325 Sept 2010)

9. L Zhang, K Zheng, W Wang, L Huang, Performance analysis on carrier scheduling schemes in the long-term evolution-advanced system with carrier aggregation. IET Com-mun. 5(5), 612-619 (2011)

10. G Galaviz, DH Covarrubias, A Andrade, On a spectrum resource organization strategy for scheduling time reduction in carrier aggregated systems. IEEE Commun Lett. 15(11), 1202-1204 (2011)

11. S Masashige, M Akihito, M Nobuhiko, Performance evaluation of inter-cell interference coordination and cell range expansion in heterogeneous networks for LTE-Advanced downlink, in Wireless Communication Systems (ISWCS), 2011 8th International Symposium on, Aachen, Germany, pp. 844-848 (6-9 Nov 2011)

12. C Mehlführer, M Wrulich, JC Ikuno, D Bosanska, M Rupp, Simulating the long term evolution physical layer, in 17th European Signal Processing Conference (EUSIPCO 2009), Glasgow, Scotland, (24-28 Aug 2009)

13. International Telecommunicaition Union, Guidelines for evaluation of radio interface technologies for IMT-Advanced, Report ITU-R M.2135-1 (Dec 2009)

14. F Meucci, O Cabral, FJ Velez, A Mihovska, NR Prasad, Spectrum aggregation with multi-band user allocation over two frequency bands, in Mobile WiMAX Symposium, 2009. MWS '09,IEEE, Napa Valley, California, USA, pp. 81-86 (9-10 July 2009)

\section{Submit your manuscript to a SpringerOpen ${ }^{\mathcal{O}}$ journal and benefit from:}

- Convenient online submission

- Rigorous peer review

- Immediate publication on acceptance

- Open access: articles freely available online

- High visibility within the field

- Retaining the copyright to your article

Submit your next manuscript at $\boldsymbol{\nabla}$ springeropen.com 